\title{
Reliability Analysis of Two Unit Standby Model with Controlled and Uncontrolled Failure of Unit and Replacement Facility Available in the System
}

\author{
Nafeesa Bashir $^{1 *}$, JPS Joorel², TR Jan ${ }^{3}$
}

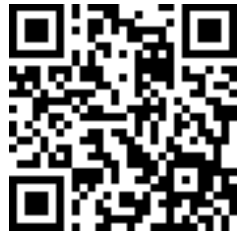

* Corresponding Author

1. Department of Statistics, University of Kashmir, India, nafeesabashir8@gmail.com

2. Department of Statistics, University of Jammu, India, joorel@ rediffmail.com

3. Department of Statistics, University of Kashmir, India, drtrjan@ gmail.com

\begin{abstract}
Planning a highly reliable and efficient system has always been a primary interest for reliability engineers by devising the powerful design strategy and employing effective repair and replacement policy. Keeping in view this, the basic aim of this paper is to analyze the reliability of a system which comprised of two units A and B in which unit A is functional and B is held standby. Unit A after failure may be controlled or uncontrolled. The failed unit undergoes for repair in the controlled unit. If repair of a unit is not controlled then it is replaced by a new one. Upon breakdown of operational unit A, unit B come becomes active instantaneously. Unit B after failure is repaired by regular repairmen. System failure takes place when both the units quit serving. The unit serves as good as a fresh after preventive repair and replacement policy. The regenerative point technique has been used to obtain the expression for several reliability measures. Finally, the graphical behavior of MTSF and profit of the present model has been observed for arbitrary values of parameters and costs.
\end{abstract}

Key Words: MTSF, Availability Analysis, Regenerative Point Technique.

\section{Introduction:}

The Scientific achievements and ever-increasing urge of the society is making the design of system more complicated. As such designing reliable models has become center attention for reliability professionals. Repairing of such systems turns out to be the crucial matter in reliability theory. Since the complete control of system failures is bizarre but their strength and effectiveness can be controlled by making use of some costeffective design approach and using suitable repair and replacement policies. Hence the main objective of the reliability experts is to frame the model more productive and profitable.

Various researchers have done a lot of work in the field of reliability on stochastic modelling with different types of failure, repair and replacement strategies. Cao et al. (1989) obtained reliability analysis of two unit model with replaceable repair facility in the system. Kadyan et al. (2010) carried out the cost benefit analysis of the model with priority to repair and degradation. Kumar and Malik (2011) obtained profit analysis of computer system and gave priority to software replacement subject to maximum operations and repair times. Further, Suriya et al. (2012) analyzed computer system with priority to software replacement over hardware replacement. Bhardwaj and Singh (2014) studied behavior of a cold-standby system with repair and replacement facility available in the system. Bashir, Joorel and Kour (2016) investigated controlled and uncontrolled demand factor and inspection in the system. Recently, Barak and Yadav (2016) developed a cold standby system with server failure. Barak et al. (2017a, 2017b) studied stochastic analysis of two-unit redundant system with priority to inspection over repair. Gitanjali and Malik (2018) analyzed the system performance by using notion of repair and maintenance of the failed unit. Poonam and Malik (2018) stochastically carried out the reliability analysis of parallel system. Barak et al. (2018) developed a two-unit system with standby and server failure subject to inspection. Kumar, Pawar and Malik (2019) studied weathering server system giving priority to repair of main unit. Kumar, Kumar and Saini (2019) obtained the performance of computer system with highest operational time.

Hence to support the existing literature and to sustain a requisite level of reliability, here a two-unit system model has been examined stochastically with the notion of replacement and repair policy. The system consists of two Units, A and B. Unit A is active while as unit B is kept as standby. The failure of the system 
may be either controlled or uncontrolled. If failure of the unit A is controlled then usual repairperson attends the system to do the repair activities of the failed unit. However if the breakdown of unit A is uncontrolled then unit A is replaced by new one. Moreover unit B after failure is repaired by regular repairperson. The system may work as fresh as new after getting repaired and replaced. Further the failure time distributions of both the units are taken as exponential. Also, the times to repair distribution of both the units are assumed as general.

\section{Notations and Symbols:}

$\theta_{1}=$ failure rate of unit $\mathrm{A}$ when $\mathrm{A}$ is in controlled state.

$\theta_{2}=$ failure rate of unit $\mathrm{A}$ when $\mathrm{A}$ is in uncontrolled state.

$\gamma_{1}=$ replacement rate of $A$.

$\alpha_{1}=$ failure rate of $\mathrm{B}$.

$H(x)=$ repair rate of $\mathrm{A}$ when $\mathrm{A}$ is in controlled state.

$G(x)=$ repair rate of $\mathrm{A}$ when $\mathrm{A}$ is in uncontrolled state.

$M(x)=$ repair rate of $\mathrm{B}$.

$A_{0}=$ Unit $\mathrm{A}$ is normal and operative.

$B_{0}=$ Unit B is normal and operative.

$B_{S}=$ Unit B is standby.

$A_{r}^{c}=$ Unit A fails, which is controllable and under repair.

$A_{r}^{u c}=$ Unit A fails, and is under repair.

$A_{\text {rep }}=$ Unit $\mathrm{A}$ is in failure mode and is under replacement.

$B_{w r}=$ Unit B waits for repair.

\section{States of the System:}

The possible states of the system are:

$$
\begin{array}{lc}
S_{0}=\left[A_{0}, B_{s}\right] & S_{1}=\left[A_{r}^{u c}, B_{0}\right] \\
S_{2}=\left[A_{r}^{c}, B_{0}\right] & S_{3}=\left[A_{r}^{c}, B_{w r}\right] \\
S_{4}=\left[A_{0}, B_{r}\right] & S_{5}=\left[A_{r e p}, B_{0}\right] \\
S_{6}=\left[A_{r}^{u c}, B_{w r}\right] & S_{7}=\left[A_{r e p}, B_{w r}\right]
\end{array}
$$




\section{TRANSITION DIAGRAM}
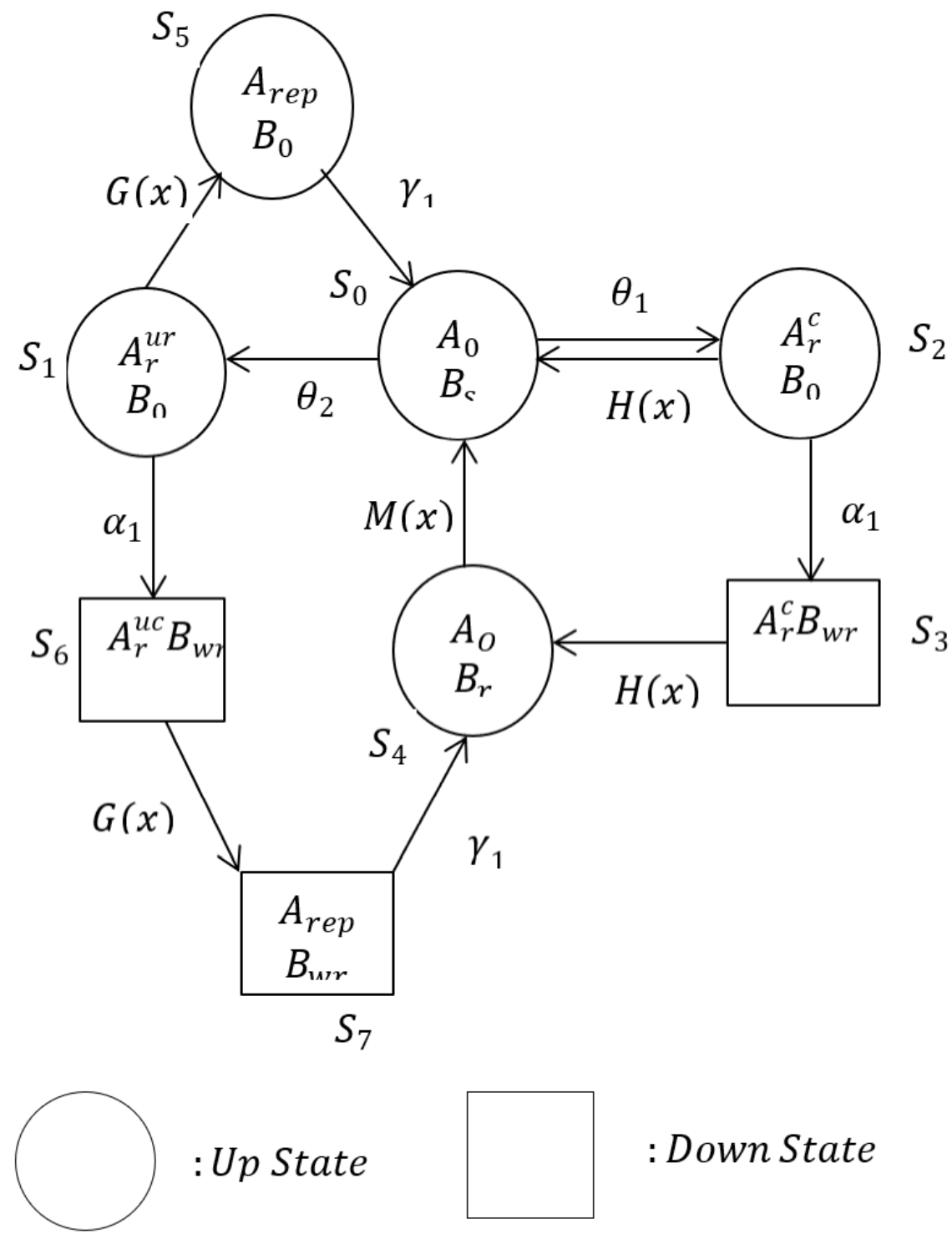

: Down State

\section{System Description:}

The system can be in any of the following states under given assumptions:

State 0: Initially unit A is operational and unit B is kept standby.

State 1: Unit A is in failed state (uncontrolled), it goes for repair while Unit B becomes operational and the system is working and hence in upstate.

State 2: Upon A is in failure mode (controllable) and is under repair. Unit B comes in operation and the system is in upstate.

State 3: Unit A is in failure mode and is continuously under repair from past state. Unit B has also failed and is waiting for repair. The system is in down state.

State 4: Unit A is in operational mode. Unit B has failed and repairman is busy in repairing the failed unit.

Stage 5: The uncontrolled failure unit under repair is replaced by a new unit $\left(A_{r e p}\right)$. Unit $\mathrm{B}$ comes is in operational mode. The system is in upstate.

State 6: The uncontrolled Unit A is under repair while as Unit B has also failed and is waiting for repair. The system is in down state. 
State 7: The uncontrolled Unit A is replaced by a new unit. Unit B is waiting for repair. The system is in down state.

\section{Transition Probabilities:}

Let $Q_{i j}(t)$ denotes the transition probability from state i to state $\mathrm{j}$ in transient state which can be obtained as:

$$
\begin{aligned}
& Q_{01}(t)=\theta_{2} \int_{0}^{t} e^{-\left(\theta_{1}+\theta_{2}\right) u} d u \\
& Q_{02}(t)=\theta_{1} \int_{0}^{t} e^{-\left(\theta_{1}+\theta_{2}\right) u} d u \\
& Q_{15}(t)=\int_{0}^{t} e^{-\left(\alpha_{1}\right) u} d G(u) \\
& Q_{16}(t)=\alpha_{1} \int_{0}^{t} e^{-\left(\alpha_{1}\right) u} d \bar{G}(u) \\
& Q_{20}(t)=\int_{0}^{t} e^{-\left(\alpha_{1}\right) u} d H(u) \\
& Q_{23}(t)=\alpha_{1} \int_{0}^{t} e^{-\left(\alpha_{1}\right) u} \bar{H}(u) d u \\
& Q_{34}(t)=\int_{0}^{t} H(u) d u \\
& Q_{40}(t)=\int_{0}^{t} M(u) d u \\
& Q_{50}(t)=\gamma_{1} \int_{0}^{t} e^{-\left(\gamma_{1}\right) u} d u \\
& Q_{67}(t)=\int_{0}^{t} G(u) d u \\
& Q_{74}(t)=\gamma_{1} \int_{0}^{t} e^{-\left(\gamma_{1}\right) u} d u
\end{aligned}
$$

As limit $t \rightarrow \infty$ in transition probabilities, the steady state probabilities can be obtained as

$$
\begin{aligned}
& P_{01}=Q_{i j}(\infty)=\int_{0}^{\infty} Q_{i j}(t) \\
& P_{01}=\frac{\theta_{2}}{\theta_{1}+\theta_{2}} \\
& P_{02}=\frac{\theta_{1}}{\theta_{1}+\theta_{2}} \\
& P_{15}=\widetilde{G}\left(\alpha_{2}\right) \\
& P_{16}=1-\widetilde{G}\left(\alpha_{2}\right) \\
& P_{20}=\widetilde{H}\left(\alpha_{2}\right) \\
& P_{23}=1-\widetilde{H}\left(\alpha_{2}\right) \\
& P_{34}=P_{40}=P_{50}=P_{67}=P_{74}=1
\end{aligned}
$$

Here it can easily be verified that $\sum_{j} P_{i j}=1$; for all possible values of $i$.

i.e.

$p_{01}+p_{02}=1$

$p_{15}+p_{16}=1$

$p_{20}+p_{23}=1$

$P_{34}=P_{40}=P_{50}=P_{67}=P_{74}=1$

\section{Mean Sojourn Times:}

If $\mathrm{T}_{\mathrm{i}}$ represents the sojourn time in state $\mathrm{S}_{\mathrm{i}}$ then mean sojourn time $\Psi_{\mathrm{i}}$ in state $\mathrm{S}_{\mathrm{i}}$ is:

$\Psi_{\mathrm{i}}=\mathrm{E}\left[\mathrm{T}_{\mathrm{i}}\right]=\int_{0}^{\infty} P\left(T_{i}>t\right) d t$

Thus

$$
\begin{aligned}
& \Psi_{0}=\frac{1}{\theta_{1}+\theta_{2}} \\
& \Psi_{1}=\frac{1}{\alpha_{1}}\left[1-\tilde{G}\left(\theta_{2}\right)\right] \\
& \Psi_{2}=\frac{1}{\alpha_{1}}\left[1-\widetilde{H}\left(\theta_{2}\right)\right] \\
& \Psi_{3}=\int_{0}^{\infty} \bar{H}(u) \mathrm{du}=1 \\
& \Psi_{4}=\int_{0}^{\infty} \bar{M}(u) \mathrm{du}=1 \\
& \Psi_{5}=\frac{1}{\gamma_{1}} \\
& \Psi_{6}=\int_{0}^{\infty} \bar{G}(u) \mathrm{du}=1
\end{aligned}
$$


$\Psi_{7}=\frac{1}{\gamma_{1}}$

\section{Mean Time To System Failure:}

To get the expressions for MTSF denoted by $\pi_{i}(t)$ for different values of $i$, the arguments of regenerative point processes has been used, taking the Laplace transform and solving the resultant set of equations for $\pi_{0}^{*}(s)$, we have

$\tilde{\pi}_{0}(s)=\frac{N_{1}(s)}{D_{1}(s)}$

where

$N_{1}(s)=\tilde{Q}_{01}(s) \widetilde{Q}_{16}(s)+\tilde{Q}_{23}(s) \widetilde{Q}_{02}(s)$

$D_{1}(s)=1-\left(\tilde{Q}_{01}(s) \tilde{Q}_{15}(s)+\tilde{Q}_{02}(s) \tilde{Q}_{20}(s)\right)$

On taking $s \rightarrow 0$ and using the relation $\breve{Q}_{i j}(s) \rightarrow P_{i j}$, we have

$\tilde{\pi}_{0}(0)=\frac{N_{1}(0)}{D_{1}(0)}=1$

Thus $N_{1}(0)=D_{1}(0)$ showing that $\tilde{\pi}_{0}(0)=1$. Hence $\pi_{0}(t)$ is a proper cdf.

Therefore, mean time to system failure when the initial state is $S_{0}$, is given by

$E(T)=-\left.\frac{d \widetilde{\pi}_{0}(s)}{d s}\right|_{s=0}=\frac{D_{1}^{\prime}(0)-N_{1}^{\prime}(0)}{D_{1}(0)}$

where,

$D^{\prime}{ }_{1}(0)-N^{\prime}{ }_{1}(0)=\Psi_{0}+\Psi_{1} p_{01}+\Psi_{2} p_{02}$

$D_{1}(0)=1-P_{01} P_{15}-P_{02} P_{20}$

M.T.S.F $=\frac{\Psi_{0}+\Psi_{1} p_{01}+\Psi_{2} p_{02}}{1-P_{01} P_{15}-P_{02} P_{20}}$

Availability Analysis :

To obtain recurrence relations among different point wise availabilities denoted by $A_{i}(t)$, we use the simple probabilistic arguments. Taking the Laplace transform and solving the resultant set of equations for $A_{0}^{*}(s)$, we have:

$A_{0}^{*}(s)=\frac{N_{2}(s)}{D_{2}(s)}$

Where

$N_{2}(s)=\left[\Psi_{0}^{*}+q_{01}^{*} \Psi_{1}^{*}+q_{02}^{*} \Psi_{2}^{*}+\left(q_{01}^{*} q_{16}^{*} q_{67}^{*} q_{74}^{*}+q_{02}^{*} q_{23}^{*} q_{34}^{*}\right) \Psi_{4}^{*}+q_{01}^{*} q_{15}^{*} \Psi_{5}^{*}\right]$

$D_{2}(s)=\left[1-\left(q_{01}^{*} q_{15}^{*} q_{50}^{*}+q_{01}^{*} q_{16}^{*} q_{67}^{*} q_{74}^{*} q_{40}^{*}+q_{02}^{*} q_{20}^{*}+q_{02}^{*} q_{23}^{*} q_{34}^{*} q_{40}^{*}\right)\right]$

The steady state Availability will be given by

$\mathrm{A}_{0}=\lim _{\mathrm{t} \rightarrow \infty} \mathrm{A}_{0}(\mathrm{t})=\lim _{\mathrm{s} \rightarrow 0} \mathrm{~s} \mathrm{~A}_{0}^{*}(\mathrm{~s})=N_{2}(0) / D_{2}^{\prime}(0)$

Where

$N_{2}(0)=\Psi_{0}+P_{01} \Psi_{1}+P_{02} \Psi_{2}+\left(P_{01} P_{16}+P_{02} P_{23}\right) \Psi_{4}+P_{01} P_{15} \Psi_{5}$

$D_{2}^{\prime}(0)=\Psi_{0}+\Psi_{1} p_{01}+\Psi_{2} p_{02}+\Psi_{3} p_{02} p_{23}+\Psi_{4}\left(p_{01} p_{16}+p_{02} p_{23}\right)+\Psi_{5} p_{01} p_{15}+$

$\Psi_{6} p_{01} p_{16}+\Psi_{7} p_{01} p_{16}$

\section{Busy Period Analysis For Regular Repairman:}

We obtain busy period for regular repairman denoted by $B_{i}(t)$ we use probabilistic arguments, taking the Laplace transform and solving the resultant set of equations for $B_{0}^{*}(s)$,

we have

Where

$$
B_{0}^{*}(s)=N_{3}(s) / D_{2}(s)
$$

$N_{3}(s)=\quad\left[q_{01}^{*} \Psi_{1}^{*}+q_{02}^{*} \Psi_{2}^{*}+q_{02}^{*} q_{23}^{*} \Psi_{3}^{*}+\left(q_{01}^{*} q_{16}^{*} q_{67}^{*} q_{74}^{*}+q_{02}^{*} q_{23}^{*} q_{34}^{*}\right) \Psi_{4}^{*}+q_{01}^{*} q_{15}^{*} \Psi_{5}^{*}+q_{01}^{*} q_{16}^{*} \Psi_{6}^{*}+\right.$ $\left.q_{01}^{*} q_{16}^{*} q_{67}^{*} \Psi_{7}^{*}\right]$

$D_{3}(s)=D_{2}(s)$ is same as in availability analysis which is given by (1)

In the steady state, the probability that the regular repairman will be busy is given by

$B_{0}=\lim _{t \rightarrow \infty} B_{0}(t)=\lim _{s \rightarrow 0} s B_{0}^{*}(s)=N_{3}(0) / D_{2}^{\prime}(0)$

Where $N_{3}(0)=P_{01} \Psi_{1}+P_{01} P_{15} \Psi_{5}+P_{01} P_{16} \Psi_{6}+P_{01} P_{16} \Psi_{7}+\left(P_{01} P_{16}+P_{02} P_{23}\right) \Psi_{4}+$ $P_{02} \Psi_{2}+P_{02} P_{23} \Psi_{3}$ 
and $D_{2}^{\prime}(0)$ is same as in the case of availability given by (4)

\section{Expected Number Of Visits By Regular Repairman:}

We obtain the expected number of visits by regular repairman denoted by $V_{i}(t)$ by using probabilistic arguments, taking the Laplace transform and solving the resultant set of equations for $\widetilde{V}_{0}(s)$, we get

$\tilde{V}_{0}(s)=\frac{N_{4}(s)}{D_{2}(s)}$

Where,

$N_{4}(s)=q_{01}^{*}+q_{02}^{*}$.

$D_{4}(s)=D_{2}(s)$ is similar as in availability analysis given by (1)

In steady state, number of visits per unit time is given by

$V_{0}(0)==\lim _{t \rightarrow \infty} \frac{V_{0}(t)}{t}=\frac{N_{4}(0)}{D_{2}^{\prime}(0)}$

Where $N_{4}(s)=P_{01}+P_{02}=1$

Profit Analysis:

The expected uptime, down time of the system and busy period and number of visits of the repairman in $(0, t]$ are given as:

$\mu_{u p}=\int_{0}^{t} A_{0}(u) d u$

$\mu_{d n}(t)=1-\mu_{u p}(t)$

$\mu_{b}(t)=\int_{0}^{t} B_{0}(u) d u$

$\mu_{v}(t)=\int_{0}^{t} V_{0}(u) d u$

so that,

$\mu_{u p}^{*}(s)=A_{0}^{*}(s) / s$

$\mu_{d n}^{*}(s)=1 / \mathrm{s}^{2}-\mu_{u p}^{*}(s)$

$\mu_{b}^{*}=B_{0}^{*} / s$

$\mu_{v}^{*}=V_{0}^{*} / s$

The expected profits incurred in $(0, t]$ can be given as $P_{1}=K_{0} A_{0}-K_{1} B_{0}-K_{2} V_{0}[15]$.

$\mathrm{K}_{0}=$ Revenue per unit up time of the system.

$\mathrm{K}_{1}=$ Cost per unit time for which the repair is busy.

$\mathrm{K}_{2}=$ Cost per unit visits by the repairman.

\section{Graphical Study of the System Model:}

In this section, the behavior for MTSF and profit function has been studied with respect to failure and repair rates. Giving the arbitrary values to the parameters the numerical results for MTSF and profit function are obtained to show the graphical behavior with respect to different parameters, keeping other value of parameters fixed. The behavior of measures is shown in Fig. 1,2,3,4.

Fig. 1 gives the graphical behavior of MTSF with respect to failure rate $\theta_{1}$, keeping the other values of parameters $\theta_{2}, \beta_{1}, \beta_{2}, \alpha_{1}, \gamma_{1}$ fixed. It is seen that as failure rate $\theta_{1}$ increases the MTSF of the system decreases. So we infer that with decrease in the failure rate of the unit which is in failure mode $\theta_{1}$, the expected life of the system increases. Fig. 2 demonstrates MTSF v/s Repair rate. We plot MTSF with respect to $\beta_{1}$ and fixed values of parameters $\theta_{1}, \theta_{2}, \beta_{2}, \alpha_{1}, \gamma_{1}$. We see that MTSF of the system increases as repair rate increases. So we conclude that with increase in repair rate of a unit in the repair mode $\beta_{1}$, the expected life of the system increases. Fig. 3 depicts the graphical behavior of profit with respect to failure rate $\theta_{1}$. It is examined that profit of the system decreases as failure rate increases regardless of other parameters. So we see that with the decrease in failure rate of unit in failure mode $\theta_{1}$, the expected life of the system increases. In Fig. 4, we plot profit with respect to repair rate $\beta_{2}$. It is clear from the graph that there is an increase in the profit of the system with increase in the repair rate regardless of the other parameters. So we infer that with increase in repair rate of the unit in repair mode $\beta_{2}$, the expected life of the system increases. 


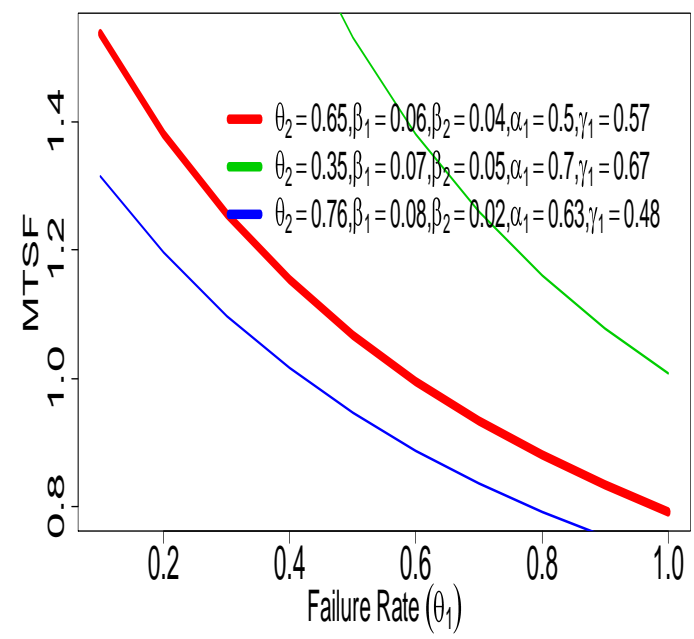

Fig. 1. Behaviour of MTSF w.r.t $\theta_{1}$ for different values of $\theta_{2}, \beta_{1}, \beta_{2}, \alpha_{1}, \gamma_{2}$

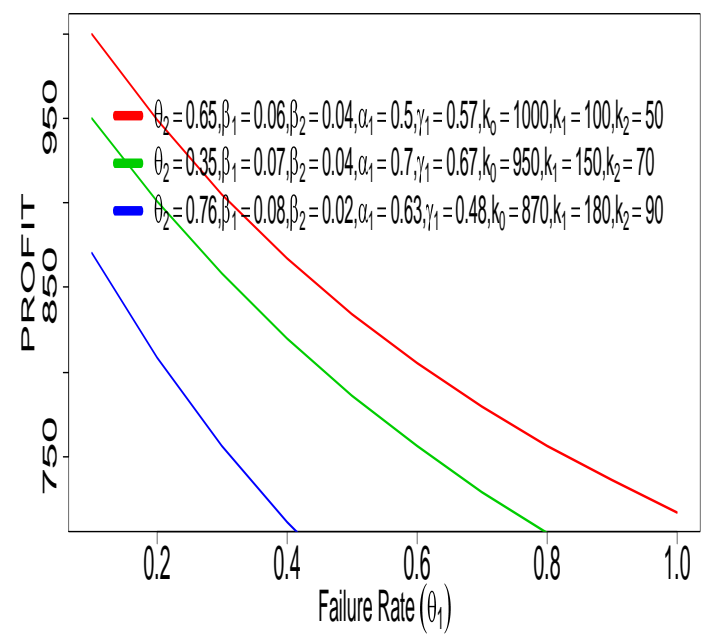

Fig. 3. Behaviour of Profit w.r.t $\theta_{1}$ for different values of $\theta_{2}, \beta_{1}, \beta_{2}, \alpha_{1}, \gamma_{1}, \mathrm{k}_{0}, \mathrm{k}_{1}, \mathrm{k}_{2}$

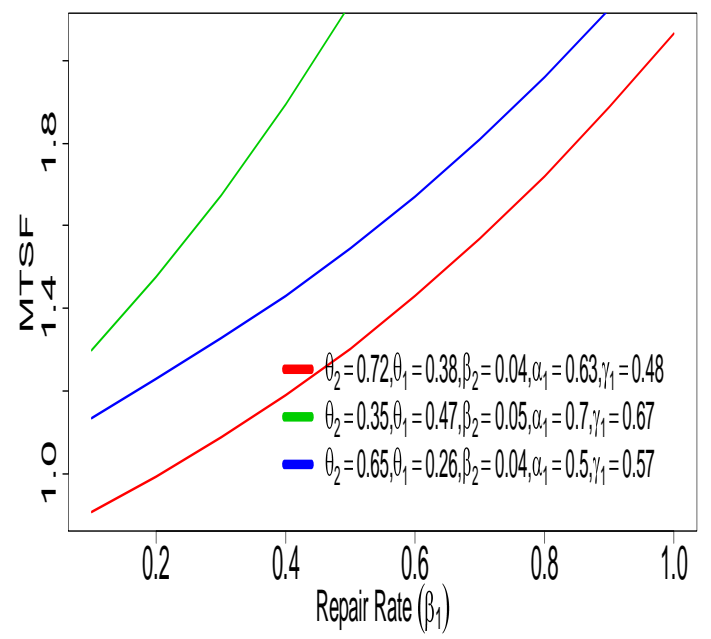

Fig. 2. Behaviour of MTSF w.r.t $\beta_{1}$ for different values of $\theta_{2}, \theta_{1}, \beta_{2}, \alpha_{1}, \gamma_{2}$

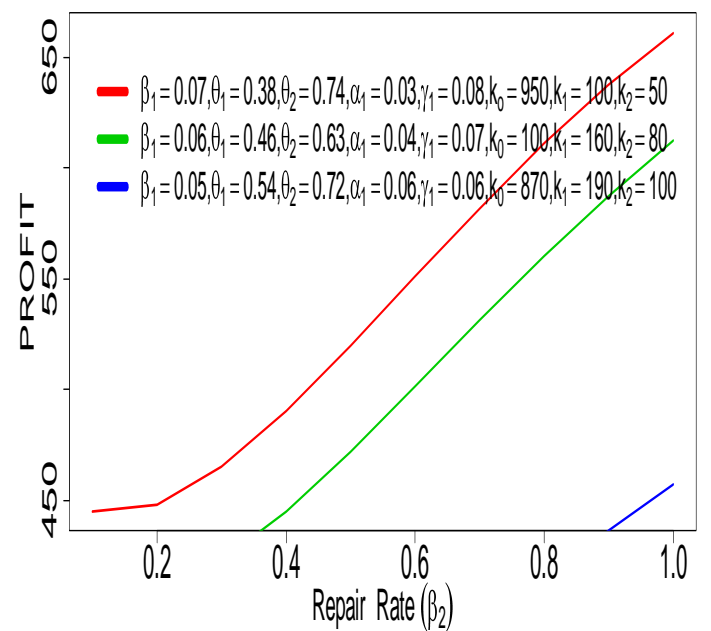

Fig. 4. Behaviour of Profit w.r.t $\beta_{1}$ for different values of $\theta_{2}, \theta_{1}, \beta_{2}, \alpha_{1}, \gamma_{1}, \mathrm{k}_{0}, \mathrm{k}_{1}, \mathrm{k}_{2}$

\section{Conclusion:}

It can be observed that with increase in the failure rate of the system, the MTSF, profit analysis of the system decreases uniformly regardless of other parameters fixed. However, we see that MTSF and profit analysis increases with increase in repair rates. Hence the study winds up that the system is more profitable by decreasing failure rate of the system in failed state and speeding up the repair rate of the unit of the system in repair mode which will in turn enhance the reliability and consequently efficiency of the system.

\section{References}

1. Barak, M. S.,Yadav, D. \& Kumari, S. (2018). "Stochastic analysis of a two-unit system with standby and server failure subject to inspection”, Life Cycle Reliab Saf Eng., pp.7:23. 2. Barak, M.S. and Yadav, D. (2016). "Stochastic analysis of a cold standby system with server failure", Int J Math Stat Invent (IJMSI), vol. 4(6) pp.18-22. 
3. Barak, M.S., Yadav, D. \& Barak, S.K. (2017a). "Stochastic analysis of a cold standby system with conditional failure of server”, Int J Stat Reliab Eng., vol. 4(1) pp. 65-69.

4. Barak, M.S., Yadav, D. \& Barak, S.K. (2017b). "Stochastic analysis of two unit redundant system with priority to inspection over repair", Life Cycle Reliab Safety Eng., https ://doi.org/10.1007/s4187 2-018-0041-0.

5. Bashir, R., Joorel, J.P.S. \& Kour, R. (2016) "Probabilistic analysis of single unit model with controlled and uncontrolled demand factor and inspection policy available in the system", International Journal of Computational and Theoretical Statistics, Vol. No. 3(1), pp. 29-38.

6. Bhardwaj., Singh,R.K. \& Ravinder (2014). "Steady state Behavior of a cold-standby system with Server failure and Arbitrary Repair, Replacement and treatment ", International journal of Applied Engineering Research, Vol. 9(24), 26563-26578 .

7. Cao, Jinhua \& Hong, W.Y.(1989), "Reliability of two-unit cold standby system with replaceable repair facility", Microelectronics Reliability, Vol. 29(2), pp. 145-150.

8. Gitanjali, and Malik,S.C. (2018) "Stochastic behavior of parallel system with expert repair and maintenance", Society for Reliability and Safety (SRESA), https://doi.org/10.1007/s41872-018-0064-6.

9. Kadyan, M.S., Malik, S.C. \& Kumar, J. (2010). " Cost analysis of a system under priority to repair and degradation", Int. J. Stat. Syst., Vol. 5(1), pp. 1-10.

10. Kumar, A. \& Malik, S.C. (2011). "Profit analysis of a computer system with priority to software replacement over hardware repair subject to maximum operation and repair times”, Int J Eng Sci Technol, Vol. 3(10), 7452-7468.

11. Kumar,A., Pawar,D. \& Malik,S.C. (2019) "Weathering Server System with Non-identical Units and Priority to Repair of Main Unit", Jour of Adv Research in Dynamical and Control Systems, Vol. 11, pp.10-18.

12. Kumar,I., Kumar,A. \& Saini,M. (2019) "Analysis of performance measures of computer systems with priority and maximum operation time, information and communication technology for sustainable development," Advances in Intelligent Systems and Computing, https://doi.org/10:1007/978-981-13-7166-0-1.

13. Malik,S.C. \& Poonam, (2018) "Stochastic analysis of a parallel system with failure of service facility", Mathematical Sciences Research Journal, 8(2), pp. 97-106.

14. Sureria,J.K., Malik, S.C. \& Anand, J. (2012)." Cost-benefit analysis of a computer system with priority to S/w replacement over H/w repair", Appl. Math. Sci., Vol. 6(75), 3723-3734. 